Simon R. Mucha, MD

Department of Critical Care Medicine, Respiratory

Institute, Cleveland Clinic; Clinical Instructor,

Cleveland Clinic Lerner College of Medicine of Case

Western Reserve University, Cleveland, $\mathrm{OH}$
NurJehan Quraishy, MD

Section Head Transfusion Medicine,

Department of Clinical Pathology, Cleveland Clinic;

Assistant Professor, Cleveland Clinic Lerner College

of Medicine of Case Western Reserve University,

Cleveland, $\mathrm{OH}$

\title{
Convalescent plasma for COVID-19: Promising, not proven
}

\section{ABSTRACT}

While promising, convalescent plasma remains experimental and is not proven effective for COVID-19. In addition, many questions remain regarding the accuracy and predictive value of antibody testing of donors and patients, optimal donor selection, optimal timing, and selection of patients most likely to benefit. Until these questions are answered, convalescent plasma should ideally be used in the context of well-designed clinical trials.

\section{KEY POINTS}

Transfusion of convalescent plasma may benefit patients with acute COVID-19 by a direct antiviral effect and possible nonspecific anti-inflammatory properties.

Convalescent plasma is likely most effective when given early in the course of the disease.

The ideal donor has high titers of neutralizing antibodies against SARS-CoV-2, although optimal testing for these antibodies is not yet established.

Convalescent plasma has been used for over a century, is likely safe, and observational data from the Expanded Access Program and limited cohort studies suggest it may be beneficial.

While convalescent plasma has received emergency use authorization from the US Food and Drug Administration, its effectiveness has yet to be established in well-controlled clinical trials, which are ongoing.

The authors report no relevant financial relationships which, in the context of their contributions, could be perceived as a potential conflict of interest.

doi:10.3949/ccjm.87a.ccc056
C OVID-19 convalescent plasma is plasma collected from donors who have recently recovered from acute COVID-19 infection. This plasma is likely to contain high levels of neutralizing antibodies against the SARS$\mathrm{CoV}-2$ virus, which, when transfused to patients with acute COVID-19 infection, can confer a degree of passive immunity.

Convalescent plasma has been used for over a century as treatment and postexposure prophylaxis for various infections. Case series from prior viral outbreaks suggest it can reduce viral load and cytokine levels and may improve clinical outcomes. Clinical trials to assess its effectiveness for the treatment of COVID-19 are ongoing.

\section{MECHANISM OF ACTION AND POTENTIAL SIDE EFFECTS}

The presumed mechanism of action of convalescent plasma is through direct binding and inactivation of the SARS-CoV-2 virus by anti-SARS-CoV-2 neutralizing antibodies. Antibody-dependent complement activation, cytotoxicity, and phagocytosis may also contribute to the therapeutic effect of neutralizing antibodies in convalescent plasma. In addition to improved viral clearance, neutralizing and nonneutralizing antibodies may also lessen disease severity and facilitate recovery by modulating the exaggerated immune response - the cytokine storm-associated with severe disease and multiorgan failure. ${ }^{1-4}$

Convalescent plasma differs from standard plasma only in that it contains anti-SARSCoV-2 antibodies. The risk of transfusionrelated adverse events is therefore likely identical to the risk associated with standard plasma, namely, transfusion-associated circu- 
latory overload, transfusion-related acute lung injury, and allergic reactions. ${ }^{5}$

An increased risk of thrombotic events has previously been reported with treatment with hyperimmune immunoglobulin. ${ }^{6}$ COVID-19 is a highly prothrombotic disease, and the impact of plasma transfusion on the coagulation system and the rate of thrombotic complications in COVID-19 is unknown.

Theoretical risks unique to anti-SARS$\mathrm{CoV}-2$ antibodies within convalescent plasma are antibody-dependent enhancement of infection and attenuated immune response with increased risk of future infection.

Antibody-dependent enhancement of infection is a phenomenon in which the presence of antibodies exacerbates the severity of the current infection. It has been well described for other viral infections such as dengue fever, and is usually due to prior infection with a virus of a different serotype. A proposed mechanism is that nonneutralizing antibodies bound to the virus surface facilitate viral entry into host cells by anchoring the virus to the host cell through host cell receptors to the $\mathrm{Fc}_{\mathrm{c}}$ portion of the antibody.

Antibody-dependent enhancement of infection has been cited as a potential reason for regional differences in severity of illness of COVID-19, but evidence for it in coronavirus infection stems mostly from in vitro studies. It is unclear if this is truly contributing to the clinical manifestation of COVID-19 or if it is relevant to treatment with convalescent plasma with high titers of neutralizing antibodies. Unfortunately, currently available antibody tests lack accuracy to determine if the SARSCoV-2 antibodies present in convalescent plasma are truly neutralizing in vivo.,

Convalescent plasma may also blunt the recipient immune response and lead to decreased formation of anti-SARS-CoV-2 antibodies, leaving patients at potentially increased risk for future infections. ${ }^{5}$

\section{HISTORICAL PRECEDENCE}

Convalescent plasma or "serum therapy" has a storied history dating back to 1901, when Emil Adolf von Behring was awarded the first Nobel Prize in medicine for its use in treating diphtheria. It was the only targeted therapy for acute infections until the advent of antibiotics in the 1940s and was used to treat various bacterial infections from pneumonia to meningitis and botulism, as well as viral infections such as mumps, measles, polio, and influenza. ${ }^{1,2}$

A meta-analysis of 8 studies involving 1,703 patients from the 1918-1920 H1N1 influenza outbreak concluded, despite many methodologic limitations, that patients treated with convalescent plasma may have experienced a clinically significant reduction in the risk of death. ${ }^{8}$

More recently, during the 2009-2010 influenza H1N1 pandemic, the use of convalescent plasma or hyperimmune globulin from convalescent plasma to treat critically ill patients was reported to be associated with improved viral clearance and decreased cytokine levels, particularly those of inflammatory cytokines. Subgroup analysis of patients treated within 5 days of disease onset showed higher survival rates with convalescent plasma-derived hyperimmune globulin than with placebo. ${ }^{9,10}$

Convalescent plasma was also used in the 2013 West African Ebola epidemic and in the 2 Ebola patients transferred to the United States (both of whom survived). ${ }^{1,2}$

Evidence supporting the use of convalescent plasma to treat coronavirus-associated disease comes from the outbreaks of SARSCoV-1 in 2003 and Middle East respiratory syndrome (MERS) in 2012. The largest study ${ }^{11}$ involved 80 critically ill patients treated with convalescent plasma during the SARS-CoV-1 outbreak in 2003 in Hong Kong. Compared with control patients (who were offered convalescent plasma but declined to give consent for experimental treatment), those who received it were reported to have higher rates of "good outcomes" if treated within 14 days of hospital admission. A good outcome was defined as being alive and discharged from the hospital by day 22 .

A meta-analysis ${ }^{12}$ of 32 studies in patients with SARS or severe influenza concluded, despite weak evidence, that convalescent plasma treatment led to statistically significant reduction in the pooled odds of mortality (odds ratio [OR] 0.25, 95\% confidence interval [CI] $0.14-0.45)$.
In 1901, von Behring won the first Nobel Prize in medicine for work on convalescent plasma 


\section{USE IN THE UNITED STATES}

Given the extraordinary circumstances of this global pandemic and the lack of effective treatment, the US Food and Drug Administration (FDA) initially allowed the use of convalescent plasma as an investigational product through 3 pathways:

- Clinical trials

- The Expanded Access Program, active from April 1 through August 31, 2020

- A single-patient emergency investigational new drug application. This option allowed patients unable or ineligible to participate in clinical trials or the Expanded Access Program to receive convalescent plasma for "serious or immediately life-threatening" COVID-19 infections under Title 21, Code of Federal Regulations 312.310, from a licensed physician upon FDA authorization. ${ }^{13}$ This option also ended August 31.

\section{Plasma collection and donor selection}

Convalescent plasma is collected by registered and licensed blood establishments that collect plasma, such as the American Red Cross. Once manufactured, it is distributed by blood centers for investigational use.

All donors must meet standard blood donation eligibility requirements and are tested for relevant transfusion-transmissible infections. The FDA ${ }^{13}$ has set the following criteria for COVID-19 convalescent plasma donors:

Laboratory confirmation of SARS-CoV-2 infection, either by nasopharyngeal polymerase chain reaction (PCR) testing at the time of illness, or a positive serologic test for SARS-CoV-2 antibodies after recovery, if PCR was not performed at the time COVID-19 was clinically suspected.

Complete resolution of symptoms at least 14 days before the donation. A negative result for COVID-19 by a diagnostic test is not necessary.

Male donors, or female donors who have never been pregnant or who have tested negative for human leukocyte antigen antibodies since their most recent pregnancy.

Testing for SARS-CoV-2 neutralizing antibodies has not been a requirement, but samples from each unit of convalescent plasma are stored for future testing once reliable antibody testing is available.
Based on studies of antibody kinetics showing immunoglobulin $\mathrm{G}$ seroconversion around day 10 and peak antibody titers around day 28, the optimal timing for convalescent plasma donation appears to be approximately 4 weeks after symptom onset. Older, male patients with more severe illness appear to develop higher antibody titers than those with minimal symptoms and may be more suitable donors. ${ }^{14-16}$

If antibody titers are available, the FDA suggests the viral neutralizing antibody titers should be at least 1:160, but titers of 1:80 are considered acceptable if an alternative matched unit is not available.

However, assays to determine viral neutralizing antibody titers are not widely available, in part because they are labor-intensive and require a biosafety level 3 laboratory if live virus is used. Viral neutralizing titers are therefore not known for the vast majority of plasma units, and a substantial portion of convalescent plasma donors may have titers below the FDA-recommended threshold. .4,17 $^{14}$ Antibody titers determined by commercially available enzyme-linked immunosorbent assay may correlate with viral neutralizing antibody titers, but have poor specificity. ${ }^{14}$

Until reliable antibody testing is widely available, convalescent plasma is collected solely on the basis of the FDA criteria above, resulting in unpredictable and likely heterogeneous viral neutralizing antibody titers across all donations.

\section{Clinical experience} with convalescent plasma for COVID-19

To date, evidence on the effectiveness of convalescent plasma for the treatment of COVID-19 is limited to case series, ${ }^{18-21}$ small cohort studies, ${ }^{22,23}$ and data from the Expanded Access Program. ${ }^{24}$ Randomized controlled trials are currently ongoing, and the currently available data from prospective trials are minimal.

Early case reports from China described patients who were alive at the time of publication with improved viral clearance, decreased cytokine levels, improved findings on chest imaging, and stable or improved oxygenation after treatment with convalescent plasma. ${ }^{18-20}$ Similar outcomes were reported in early case series from the United States. ${ }^{21}$ 
Liu et $a^{22}$ reported stable or improved oxygenation in patients treated with convalescent plasma compared with matched controls, and a lower mortality rate with treatment for nonintubated patients (hazard ratio $0.23,95 \%$ CI 0.05 $0.98, P=.046)$, but not for intubated patients (hazard ratio $0.79,95 \%$ CI 0.22-2.79, $P=.716$ ).

In contrast, Rogers et $\mathrm{al}^{23}$ were unable to demonstrate a survival benefit for their cohort of 64 patients treated with convalescent plasma compared with a matched cohort of 177 patients treated with standard of care.

The first randomized controlled trial of convalescent plasma in COVID-19 was stopped early due to slow enrollment, as local infection rates declined thanks to strict lockdown measures in Wuhan, China. ${ }^{25}$ The study was therefore underpowered to demonstrate statistically significant differences in either the primary end point (time to clinical improvement) or secondary end points (28-day mortality rate, time to hospital discharge, and rate of negative PCR testing at 72 hours). Although not statistically significant, the results appear to signal a more favorable outcome for patients treated with convalescent plasma. ${ }^{25}$

An analysis stratified by disease severity showed that patients who did not need mechanical ventilation and did not have multiorgan failure had a shorter time to clinical improvement if given convalescent plasma than with placebo. Clinically significant improvement at day 28 was also more likely to occur in the convalescent plasma group $(91.3 \%)$ than in the control group $(68.2 \%){ }^{25}$

\section{Data from the Expanded Access Program}

While clinical trials are still ongoing (on September 28, 2020, clinicaltrials.gov listed 93 clinical trials that were recruiting patients for the use of convalescent plasma to prevent or treat COVID-19), nearly all patients who received convalescent plasma in the United States did so through the US Convalescent Plasma Expanded Access Program, created in collaboration between the FDA and Mayo Clinic. ${ }^{24}$

This registry study was designed to facilitate rapid application of convalescent plasma in clinical practice and monitor its safety. It allowed physicians treating hospitalized COVID-19 patients to register and request convalescent plasma for individual qualifying patients. The physician registering a patient was required to complete all necessary documentation including consent, patient history, posttransfusion follow-up data, and adverse event reporting in a centralized electronic database administered by Mayo Clinic. ${ }^{24}$

From April through August of this year, 14,532 physicians at 2,759 sites registered 105,785 patients, 84,639 of whom received convalescent plasma through this program by August 31.24

Safety. In the first 20,000 patients who received convalescent plasma through the Expanded Access Program, the rate of serious adverse events within 4 hours of transfusion was less than $1 \% .{ }^{26}$ Sixty-three of these events $(0.3 \%$ of all transfusions) were deaths, 13 of which were judged as related to convalescent plasma (12 possibly, 1 probably, and 0 definitely). Seventy-eight nonmortality events were reported, with 36 reports of transfusionassociated circulatory overload, 21 reports of transfusion-related acute lung injury, and 21 reports of severe allergic transfusion reaction. ${ }^{26}$

Within 7 days of completion of the transfusion, 1,247 other serious adverse events were reported, ${ }^{26}$ including 113 thromboembolic or thrombotic events, 457 sustained hypotensive events requiring intravenous vasopressor support, and 677 cardiac events. The authors note that 75 of the thrombotic or thromboembolic complications and 597 of the 643 cardiac events were judged by the treating physician to be unrelated to the plasma transfusion. ${ }^{26}$

In contrast, the incidence of transfusionrelated reactions reported in a recent matched cohort study of 64 patients ${ }^{23}$ and the randomized controlled trial in 102 patients $^{25}$ was significantly higher, at $2.8 \%$ and $2.1 \%$, respectively. This highlights the challenges in assessing transfusion-related complications in critically ill patients and differentiating them from progression of disease.

Effectiveness. The first effectiveness anal$\mathrm{ysis}^{27}$ of 35,322 patients treated with convalescent plasma through the Expanded Access Program between April 4 and July 4 reported an overall mortality rate of $10.5 \%$ by day 7 and $24.9 \%$ by day 30 .

Subgroup analysis comparing patients treated with plasma early after diagnosis (within 3 days or less) vs late, and patients treated 
with plasma containing high vs low levels of anti-SARS-CoV-2 antibody, signal a possible benefit of early administration of plasma with high antibody titers.

In patients treated with convalescent plasma within 3 days of the diagnosis of COVID-19, the 7 -day mortality rate was $8.7 \%$ (95\% CI 8.3\%-9.2\%) compared with $11.9 \%$ $(11.4 \%-12.2 \%)$ in those who received it 4 or more days after diagnosis $(P<.001)$. A similar trend was observed in 30-day mortality (21.6\% vs $26.7 \%, P<.0001) .{ }^{27}$

Estimates of the antibody titers of the convalescent plasma transfused were available for 3,082 patients. Titers were estimated using the Ortho-Clinical Diagnostics VITROS Anti-SARS-CoV-2 immunoglobulin G (IgG) chemiluminescent immunoassay, a qualitative assay based on the sample signal-to-cut-off (S-Co) ratio, with values less than 1.0 and 1.0 or higher corresponding to negative and positive results. The authors used S-Co values to estimate relative levels of anti-SARS-CoV-2 antibodies by setting thresholds for "low" and "high" level sera based on approximately the 20 th and 80th percentiles of the distribution for the $\mathrm{S}-\mathrm{Co}$ ratios, respectively. ${ }^{27}$

$\lg G$ seroconversion occurs around day 10 , and titers peak around day 28
Patients who received high-IgG plasma had a lower 7-day mortality rate $(8.9 \%$ [6.8\%$11.7 \%])$ than those receiving medium- $(11.6 \%$ [10.3\%-13.1\%]) or low-IgG plasma (13.7\% [11.1\%-16.8\%]). The pooled relative risk of mortality among patients who received highantibody-level plasma units, compared with low-antibody plasma units, was 0.65 [0.47$0.92]$ at 7 days and 0.77 [0.63-0.94] at 30 days. ${ }^{27}$

This difference in relative risk of mortality at 7 days led to the now infamously retracted statement of FDA commissioner Stephen Hahn that convalescent plasma led to a 35\% improvement in survival. ${ }^{28}$

The authors of the study are more cautious in their interpretation and conclude ${ }^{27}$ that these observed "relationships between mortality and both the time to plasma transfusion and antibody levels provide a signature that is consistent with efficacy."

Limitations of the data. While these results are promising, the ability to draw definitive conclusions on efficacy is limited by the lack of a control group and heterogeneity throughout the study period.
Mortality rates for patients hospitalized for COVID-19 declined significantly over the reporting period, with overall 7-day mortality rates decreasing from $15.5 \%$ in April to $6.6 \%$ in June. This correlates with a significant decrease in severity of illness, with $49.9 \%$ of patients requiring mechanical ventilation in April compared with only $16.4 \%$ in June. ${ }^{27}$

Similarly, concomitant use of therapies that have since been proven effective or ineffective changed dramatically. For example, the number of patients in this study treated with hydroxychloroquine, which is now generally regarded as useless, declined from $62.3 \%$ in April to $1.8 \%$ in June, while the use of remdesevir, which seems to be effective, increased from $4.7 \%$ to $46.3 \% .^{27}$

Over the same time, the proportion of patients receiving low-antibody-titer plasma decreased from $26.0 \%$ to $11.9 \%$, while the proportion of patients receiving transfusions within 3 days increased from $24.7 \%$ to $50.3 \% .{ }^{25}$ The benefit in terms of lower mortality observed for early vs late plasma transfusion and transfusion with high- vs low-antibody-titer plasma may therefore simply reflect the correlation with an overall decrease in severity of illness. Again, without a well-defined control group, the data from the Expanded Access Program will not be able to answer the question of efficacy definitively.

\section{Recent studies awaiting peer review}

After the efficacy analysis of the Expanded Access Program was published, results of 3 more randomized controlled trials have been published on preprint servers. Although these publications are yet to be peer-reviewed and have some methodologic weaknesses, they raise serious doubts about the effectiveness of convalescent plasma.

Balcells et al $^{29}$ report no difference in outcomes in a single-center open-label study of immediate treatment with convalescent plasma vs delayed treatment only in case of disease progression. The early plasma group received their first plasma unit at enrollment. The deferred plasma group received convalescent plasma only if their respiratory status worsened (defined as a $\mathrm{PaO}_{2} / \mathrm{FiO}_{2}$ ratio < 200) or if the patient remained hospitalized after 7 days of enrollment with persistent symptoms. 
Fifty-eight patients were randomized, 28 in the early treatment group, and 30 in the deferred treatment group, with 13 of the 30 patients in the deferred group eventually receiving plasma due to progression of symptoms. ${ }^{29}$

There was no difference in the primary composite end point of progression to mechanical ventilation, hospitalization greater than 14 days, or in-hospital mortality, which occurred in $9(32 \%)$ of the 28 patients in the early treatment group vs $10(33 \%)$ of the 30 patients in the deferred treatment group (OR 0.95, 95\% CI 0.32-2.84). ${ }^{29}$

Patients who received plasma early had overall higher rates of death, which occurred in $5(18 \%)$ of 28 vs $2(7 \%)$ of 30 patients, OR 3.04, 95\% CI 0.54-17.2) and need for mechanical ventilation: $5(18 \%)$ of 28 vs 2 (6.7\%) of 30 patients, OR 3.04, 95\% CI 0.5417.2 , although the differences were not statistically significant. ${ }^{29}$

The Convalescent Plasma for COVID (ConCOVID) study, ${ }^{30}$ a multicenter openlabel randomized clinical trial in the Netherlands, was halted early after 53 of 66 patients tested were found to have high titers of SARS. $\mathrm{CoV}-2$ neutralizing antibodies at the time of enrollment, before they received convalescent plasma. Analysis of the available outcomes data of the total of 86 enrolled patients showed no difference in disease severity, hospital length of stay or mortality rates between patients who received plasma and the control group.

The PLACID trial, ${ }^{31}$ an open-label, multicenter randomized controlled trial comparing convalescent plasma to standard of care, enrolled 464 patients with moderate severity of illness $\left(\mathrm{PaO}_{2} / \mathrm{FiO}_{2}\right.$ ratio 200-300, or respiratory rate $>24$ per minute and $\mathrm{SpO}_{2} \leq$ 93\% on room air) in 39 centers across India. Although it demonstrated a statistically significant greater absolute decrease in $\mathrm{FiO}_{2}$ needed by day 7 (11\% vs $9.5 \%)$ and greater rate of viral clearance as demonstrated by negative SARS-CoV-2 PCR testing (67.9\% vs $54.6 \%$ ), the study showed no difference in the primary composite outcome of progression to severe disease (defined as a $\mathrm{PaO}_{2} / \mathrm{FiO}_{2}$ ratio $<100$ ) or death by day 28 , which occurred in $44(18.7 \%)$ of 235 in the treatment group vs $41(17.9 \%)$ of 229 in the standard care group, OR 1.09, 95\% CI 0.67-1.77. In- terestingly, the study reported 3 deaths that were considered to be possibly directly related to convalescent plasma transfusion $(1.3 \%$ of the active treatment group).

\section{EVIDENCE IS SUGGESTIVE, BUT WEAK}

Taken together, the evidence available today suggests treatment with convalescent plasma may improve viral clearance, decrease inflammation, and improve oxygenation, which may translate into a lower mortality rate for select patients. This treatment appears to be of greatest benefit if plasma with high titers of neutralizing antibodies is given early in the course of the disease in patients without advanced organ failure, such as respiratory failure requiring mechanical ventilation.

The evidence supporting convalescent plasma, however is weak, and serious questions remain about optimal timing, patient selection, dosing, and antibody testing of donors and patients. True safety and efficacy have yet to be confirmed in ongoing well-controlled prospective trials

Both the Infectious Diseases Society of America $^{32}$ and the National Institutes of Health Treatment Guideline Panel ${ }^{33}$ therefore concluded there is currently not enough evidence to recommend convalescent plasma as the standard of care and recommend its use in prospective, well-controlled, randomized trials.

Despite these limitations and concerns, the FDA determined that on the basis of these data the "known and potential benefits of the product, when used to treat COVID-19, outweigh the known and potential risks of the product" and has granted emergency use authorization. ${ }^{34}$ This finding authorizes the distribution and administration of COVID-19 convalescent plasma for the treatment of confirmed or suspected COVID-19. It requires that a fact sheet providing information of dosing and potential side effects be made available to patients treated with convalescent plasma and that healthcare providers maintain records, conduct a thorough investigation, and report adverse reactions and fatalities related to convalescent plasma transfusion, as required under Title 21, Code of Federal Regulations 606.170.

\section{To date, evidence is limited for the effectiveness of convalescent plasma for COVID-19}




\section{REFERENCES}

1. Casadevall A, Dadachova E, Pirofski L. Passive antibody therapy for infectious diseases. Nat Rev Microbiol 2004; 2(9):695-703. doi:10.1038/nrmicro974

2. Casadevall A, Pirofski L-A. The convalescent sera option for containing COVID-19. J Clin Invest 2020; 130(4):1545-1548. doi:10.1172/JCI138003

3. Blanco-Melo D, Nilsson-Payant BE, Liu WC, et al. Imbalanced host response to SARS-CoV-2 drives development of COVID-19. Cell 2020; 181(5):1036-1045.e9. doi:10.1016/j.cell.2020.04.026

4. Rojas M, Rodriguez Y, Monsalve DM, et al. Convalescent plasma in COVID-19: possible mechanisms of action. Autoimmun Rev 2020 19(7).102554. doi:10.1016/j.autrev.2020.102554

5. Bloch EM, Shoham S, Casadevall A, et al. Deployment of convalescent plasma for the prevention and treatment of COVID-19. J Clin Invest 2020; 130(6):2757-2765. doi:10.1172/JCI138745

6. Menis M, Sridhar G, Selvam N, et al. Hyperimmune globulins and same-day thrombotic adverse events as recorded in a large healthcare database during 2008-2011. Am J Hematol 2013; 88(12):10351040. doi:10.1002/ajh.23559

7. Kadkhoda K. COVID-19: are neutralizing antibodies neutralizing enough? Transfusion 2020; 60(7):1602-1603. doi:10.1111/trf.15897

8. Luke TC, Kilbane EM, Jackson JL, Hoffman SL. Meta-analysis: convalescent blood products for Spanish influenza pneumonia: a future H5N1 treatment? Ann Intern Med 2006; 145(8):599-609. doi:10.7326/0003-4819-145-8-200610170-00139

9. Hung IFN, To KKW, Lee C-K, et al. Hyperimmune IV immunoglobulin treatment: a multicenter double-blind randomized controlled tria for patients with severe 2009 influenza A (H1N1) infection. Chest 2013; 144(2):464-473. doi:10.1378/chest.12-2907

10. Hung IFN, To KKW, Lee C-K, et al. Convalescent plasma treatment reduced mortality in patients with severe pandemic influenza $A$ (H1N1) 2009 virus infection. Clin Infect Dis 2011; 52(4):447-456. doi:10.1093/cid/ciq106

11. Cheng Y, Wong R, Soo YOY, et al. Use of convalescent plasma therapy in SARS patients in Hong Kong. Eur J Clin Microbiol Infect Dis 2005; 24(1):44-46. doi:10.1007/s10096-004-1271-9

12. Mair-Jenkins J, Saavedra-Campos M, Baillie JK, et al. The effectiveness of convalescent plasma and hyperimmune immunoglobulin for the treatment of severe acute respiratory infections of viral etiology: a systematic review and exploratory meta-analysis. J Infect Dis 2015; 211(1):80-90. doi:10.1093/infdis/jiu396

13. US Food and Drug Administration. Recommendations for investigational COVID-19 convalescent plasma. Accessed July 23, 2020. https://www.fda.gov/vaccines-blood-biologics/investigational-newdrug-ind-or-device-exemption-ide-process-cber/recommendationsinvestigational-covid-19-convalescent-plasma\#Pathways \%20for

14. Klein SB, Pekosz A, Park H-S, et al. Sex, age, and hospitalization drive antibody responses in a COVID-19 convalescent plasma donor population. medRxiv 2020 Jun 20;2020.06.26.20139063. doi:10.1101/2020.06.26.20139063

15. Li L, Tong X, Chen $\mathbf{H}$, et al. Characteristics and serological patterns of COVID-19 convalescent plasma donors: optimal donors and timing of donation. Transfusion 2020 Jul 6;10.1111/trf.15918. doi:10.1111/trf.15918

16. Lynch KL, Whitman JD, Lacanienta NP, et al. Magnitude and kinetics of anti-SARS-CoV-2 antibody responses and their relationship to disease severity. Clin Infect Dis 2020 Jul 14; ciaa979. doi:10.1093/cid/ciaa979

17. Salazar E, Kuchipudi SV, Christensen PA, et al. Relationship between anti-spike protein antibody titers and SARS-CoV-2 in vitro virus neutralization in convalescent plasma. bioRxiv 2020 Jun 9;2020.06.08.138990. doi:10.1101/2020.06.08.138990

18. Duan K, Liu B, Li C, et al. Effectiveness of convalescent plasma therapy in severe COVID-19 patients. Proc Natl Acad Sci U S A 2020; 117(17):9490-9496. doi:10.1073/pnas.2004168117

19. Zeng F, Chen X, Deng G. Convalescent plasma for patients with
COVID-19. Letter. Proc Natl Acad Sci U S A 2020; 117(23):12528. doi:10.1073/pnas.2006961117

20. Shen C, Wang Z, Zhao F, et al. Treatment of 5 critically ill patients with COVID-19 with convalescent plasma. JAMA 2020; 323(16):15821589. doi:10.1001/jama.2020.4783

21. Salazar E, Perez KK, Ashraf M, et al. Treatment of coronavirus disease 2019 (COVID-19) patients with convalescent plasma. Am J Pathol 2020 May 27;S0002-9440(20)30257-1. doi:10.1016/j.ajpath.2020.05.014

22. Liu STH, Lin H-M, Baine I, et al. Convalescent plasma treatment of severe COVID-19: a propensity score-matched control study. Nat Med 2020 Sep 15. doi:10.1038/s41591-020-1088-9

23. Rogers RA, Shehadeh F, Mylona E, et al. Convalescent plasma for patients with severe COVID-19: a matched cohort study. medRxiv 2020.08.18.2017702. doi:10.1101/2020.08.18.20177402

24. Joyner M. EAP for convalescent plasma no longer enrolling; the FDA authorized emergency use. Accessed September 29, 2020. uscovidplasma.org

25. Li L, Zhang W, Hu Y, et al. Effect of convalescent plasma therapy on time to clinical improvement in patients with severe and lifethreatening COVID-19: a randomized clinical trial. JAMA 2020 jun3;e2010044. Correction in JAMA 2020; 324(5): 519. doi:10.1001/jama.2020.10044

26. Joyner MJ, Bruno KA, Klassen SA, et al. Safety update: COVID-19 convalescent plasma in 20,000 hospitalized patients. Mayo Clin Proc 2020; 95(9):1888-1897. doi:10.1016/j.mayocp.2020.06.028

27. Joyner MJ, Senefeld JW, Klassen SA, et al. Effect of convalescent plasma on mortality among hospitalized patients with COVID-19: initial three-month experience. medRxiv 2020. doi:10.1101/2020.08.12.20169359

28. Harris J. FDA's Hahn apologizes for overselling plasma's benefits as a COVID-19 treatment. NPR August 25, 2020. Accessed September 30, 2020. https://www.npr.org/sections/healthshots/2020/08/25/905792261/fdas-hahn-apologizes-for-oversellingplasmas-benefits-as-a-covid-19-treatment

29. Balcells ME, Rojas L, Le Corre N, et al. Early anti-SARS-CoV-2 convalescent plasma in patients admitted for COVID-19: a randomized phase II clinical trial. medRxiv 2020.09.17.20196212. doi:10.1101/2020.09.17.20196212

30. Gharbharan A, Jordans CCE, Geurts van Kessel C, et al. Convalescent plasma for COVID-19. A randomized clinical trial. medRxiv 2020.07.01.20139857. doi:10.1101/2020.07.01.20139857

31. Agarwal A, Mukherjee A, Kumar G, et al. Convalescent plasma in the management of moderate COVID-19 in India: an open-label parallel-arm phase II multicentre randomized controlled trial (PLACID Trial) medRxiv 2020.09.03.20187252. doi:10.1101/2020.09.03.20187252

32. Infectious Diseases Society of America. Infectious Diseases Society of America Guidelines on the Treatment and Management of Patients with COVID-19. Accessed September 29, 2020. https://www. idsociety.org/practice-guideline/covid-19-guideline-treatment-andmanagement/\#toc-7

33. National Institutes of Health. The COVID-19 Treatment Guidelines Panel's statement on the emergency use authorization of convalescent plasma for the treatment of COVID-19. Accessed September 29, 2020. https://www.covid19treatmentguidelines.nih.gov/statementon-convalescent-plasma-eua

34. US Food and Drug Administration. FDA issues emergency use authorization for convalescent plasma as potential promising COVID-19 treatment, another achievement in administration's fight against pandemic. August 23, 2020. Accessed September 30, 2020. https:// www.fda.gov/news-events/press-announcements/fda-issues-emergency-use-authorization-convalescent-plasma-potential-promisingcovid-19-treatment

Address: NurJehan Quraishy, MD, Clinical Pathology, Q6-2, Cleveland Clinic, 9500 Euclid Avenue, Cleveland, OH 44195; quraisn@ccf.org 